\title{
INVARIANT DIFFERENTIAL OPERATORS ON A REAL SEMISIMPLE LIE ALGEBRA AND THEIR RADIAL COMPONENTS
}

\author{
BY
}

\author{
MOHSEN PAZIRANDEH( 1 )
}

ABSTRACT. Let $S\left(g_{c}\right)$ be the symmetric algebra over the complexification $\theta_{c}$ of the real semisimple Lie algebra 8 . For $u \in S\left(\theta_{c}\right), \partial(u)$ is the corresponding differential operator on $\theta \cdot D(8)$ denotes the algebra generated by $\partial\left(S\left(B_{c}\right)\right)$ and multiplication by polynomials on $s_{c}$. For any open set $U \subset \theta$, $\operatorname{Diff}(U)$ is the algebra of differential operators with $C^{\infty}$-coefficients on $U$. Let $b$ be a Cartan subalgebra of $b, b^{\prime}$ the set of its regular points and $\pi=$ $\Pi_{a \in P} a, P$ some positive system of roots. Let $W=\left(b^{\prime}\right)^{G}, G$ the connected adjoint group of $\boldsymbol{B}$.

Harish-Chandra showed that, for each $D \in \operatorname{Diff}(W)$, there is a unique differential operator $\delta_{\mathfrak{b}}^{\prime}(D)$ on $b^{\prime}$ such that $\left.(D f)\right|_{b} ^{\prime}=\delta_{b}^{\prime}(D)\left(\left.f\right|_{b}\right)$ for all $G$-invariant $f \in C^{\infty}(W)$, and that if $D \in D(b)$, then $\delta_{b}^{\prime}(D)=\pi^{-1^{\prime}} \circ \bar{D} \circ \pi$ for some $\bar{D} \in \mathcal{D}(\theta)$. In particular $\overline{\partial(u)}=\partial\left(\left.u\right|_{b}\right), u \in S\left(B_{c}\right)$ and invariant.

We prove these results by different, yet simpler methods. We reduce evaluation of $\delta_{b}^{\prime}(\partial(u))\left(u \in S\left(g_{c}\right)\right.$, invariant) via Weyl's unitarian trick, to the case of compact $G$. This case is proved using an evaluation of a family of $G$-invariant eigenfunctions on:

$$
\pi(H) \pi\left(H^{\prime}\right) \int_{G} \exp B\left(H^{x}, H^{\prime}\right) d x=c \sum_{S \in W\left(B_{c^{\prime}} h_{c}\right)} \epsilon(s) \exp B\left(s H, H^{\prime}\right),
$$

For $G$-invariant $D \in \mathscr{D}(g)$, we prove $\pi^{-1} \circ \delta^{\prime}(D) \circ \pi \in \mathscr{D}(b)$ using properties of derivations $E \rightarrow[\partial(u), E]$ of $D(\theta)$ induced by $\partial(u)\left(u \in S\left(\theta_{c}\right)\right)$ and of the algebra of polynomials on ${ }^{b} c$ invariant under the Weyl group.

1. Preliminaries. Our aim here is to give alternative proofs to some results of Harish-Chandra on radial components of invariant differential operators on a real semis imple Lie algebra [1], [2].

Let $g$ be a semisimple Lie algebra over $R, g_{c}$ its complexification, 5 a Cartan subalgebra of $g$, and $\xi_{c}$ complexification of $\mathscr{G}$. Denote by $g^{\prime}$ the set of regular elements of $g$ and $\zeta^{\prime}=\mathscr{G} \cap g^{\prime}$. We have $H \in \mathscr{G}^{\prime}$ if and only if $\pi(H) \neq 0$,

Received by the editors May 2, 1972.

AMS (MOS) subject classifications (1970). Primary 17B20; Secondary 17 B99.

Key words and phrases. Radial components, invariant differential operators.

(1) The author is greatly indebted to Professer V. S. Varadarajan, who has been of valuable help in preparation of this work. This constitutes portions of the author's Ph. D. dis sertation. 
where $\pi=\Pi_{a \in P} \alpha, P$ being a positive system of roots of $g_{c}$ with respect to $G_{c}$. Let $G$ be a connected Lie group with Lie algebra g. Let $S\left(g_{c}\right)$ and $P\left(g_{c}\right)$ be the symmetric and the polynomial algebras over $g_{c}$, and $Q\left(g_{c}\right)$ quotient field of $P\left(g_{c}\right)$. We assume as usual that $g_{c} \subset S\left(g_{c}\right)$. Let $U$ be an open subset of $g$, and $C^{\infty}(U)$ the algebra of $C^{\infty}$ functions on $U$. An element $X \in g$ acts as a derivation of $C^{\infty}(U)$ given by

$$
(X f)(u)=d f(u+t X) /\left.d t\right|_{t=0}
$$

where $f \in C^{\infty}(U), u \in U$, and $t \in \mathbf{R}$. We denote the above derivation by $\partial(X)$ and extend the map $\partial: X \mapsto \partial(X)$ uniquely to a homomorphism of $S\left(g_{c}\right)$ into the associative algebra of endomorphisms of $C^{\infty}(U)$. If $\phi \in C^{\infty}(U)$, then $\phi$ is identified with the endomorphism $\phi: f \mapsto \phi f$ of $C^{\infty}(U)$. The algebra generated by $\{\phi, \partial(p) \mid$ $\left.\phi \in C^{\infty}(U), p \in S\left(g_{c}\right)\right\}$ is denoted by Diff $(U)$. It is called the algebra of differential operators on $g$ with $C^{\infty}$ coefficients. We denote by $\mathcal{D}(\mathrm{g})$ the algebra generated by $P\left(g_{c}\right)$ $\cup \partial\left(S\left(g_{c}\right)\right)$, and refer to it as the algebra of differential operators on $g$ with polynomial coefficients. We write $f(u ; D)$ to mean $(D f)(u)$, for $D \in \operatorname{Diff}(U), u \in U, f \in C^{\infty}(U)$. For any $D \in \operatorname{Diff}(U)$, and $u \in U$, there is a unique $p \in S\left(g_{c}\right)$, such that $f(u ; D)=f(u ; \partial(p))$. $\partial(p)$ is called the local expression of $D$ at $u$, and is denoted by $D_{u}$.

Let $U$ be an open subset of $g$ invariant under $G$. $G$ acts naturally on $C^{\infty}(U)$ and $\operatorname{Diff}(U)$ if we set

$$
f^{x}(X)=f\left(X^{x-1}\right) \quad\left(x \in G, X \in U, f \in C^{\infty}(U)\right)
$$

and

$$
D^{x} f=\left(D f^{x}\right)^{x-1} \quad\left(D \in \operatorname{Diff}(U), \quad x \in G, \quad f \in C^{\infty}(U)\right)
$$

$D$ (resp. $f$ ) is called invariant under $G$ if $D^{x}=D$ (resp. $f^{x}=f$ ) for all $x \in G$. Let $l^{\infty}(U)=\left\{f: f \in C^{\infty}(U), f^{x}=f\right.$ for all $\left.x \in G\right\}$, and $\operatorname{Diff}_{\text {inv }}(U)=\{D \in \operatorname{Diff}(U)$ : $D^{x}=D$ for all $\left.x \in G\right\}$. We put $\mathscr{I}(g)=\left\{D \in \mathscr{D}(g) \mid D^{x}=D\right.$ for all $\left.x \in G\right\}$.

We now take $U=\left(\mathscr{G}^{\prime}\right)^{G}$. Then, corresponding to any $D \in \operatorname{Diff}_{\text {inv }}(U)$, HarishChandra [1] constructed a differential operator $\delta_{b}^{\prime}(D)$ on $\zeta^{\prime}$ such that

$$
f(H ; D)=\bar{f}\left(H ; \delta_{\mathfrak{b}}^{\prime}(D)\right) \quad\left(\bar{f}=\left.f\right|_{\mathfrak{b}^{\prime}}\right)
$$

for all $f \in I^{\infty}(U)$, and $H \in \mathscr{G}^{\prime} . \delta_{\mathfrak{b}}^{\prime}(D)$ is called the radial component of $D$ on $\mathscr{G}^{\prime}$ and we have the following result (Harish-Chandra [1]):

Theorem 1.1. Given any $D \in \operatorname{Diff}_{\text {inv }}\left(\left(\zeta^{\prime}\right)^{G}\right)$, there is exactly one $\delta_{\mathfrak{b}}^{\prime}(D) \epsilon$ Diff $\left(\zeta^{\prime}\right)$ such that

$$
f(H ; D)=\bar{f}\left(H ; \delta_{b}^{\prime}(D)\right) \quad\left(f \in I^{\infty}\left(\left(G^{\prime}\right)^{G}\right)\right) .
$$

The operator $\delta_{b}^{\prime}(D)$ is invariant under the normalizer of $\bar{G}$ in $G$. The map $D \rightarrow \delta_{b}^{\prime}(D)$ is a bomomorphism of $\mathrm{Diff}_{\mathrm{inv}}\left(\left(\xi^{\prime}\right)^{G}\right)$ into $\mathrm{Diff}\left(\xi^{\prime}\right)$. 
It turns out to be important to calculate $\delta_{\mathfrak{h}}^{\prime}(D)$ explicitly for at least the most important of the invariant differential operators $D$. This was first done by HarishChandra. We shall now describe two of his results. Let $I_{s}\left(g_{c}\right)$ be the algebra of $G$-invariant elements of $S\left(g_{c}\right)$, and $I_{s}\left(G_{c}\right)$ the subalgebra of $S\left(G_{c}\right)$ of elements invariant under the Weyl group $W\left(g_{c}, g_{c}\right)$. If $p \in I_{s}\left(g_{c}\right)$, then by a theorem of Chevalley, there is a unique $p_{b} \in I_{s}\left(\mathscr{G}_{c}\right)$ such that $p-p_{b}$ is in the ideal generated by the root-s paces. Harish-Chandra [1] showed that, for $p \in S\left(g_{c}\right)$,

$$
\delta_{\mathfrak{h}}^{\prime}(\partial(p))=\pi^{-1} \circ \partial\left(p_{\mathfrak{h}}\right) \circ \pi
$$

Generalizing this, he proved in [2] that, for any $D \in \mathscr{D}\left(g_{c}\right)$ which is invariant, $\pi \circ \delta_{\mathfrak{b}}^{\prime}(D) \circ \pi^{-1}$ is the restriction to $\xi^{\prime}$ of an element of $\mathcal{D}\left(\xi_{c}\right)$ that is invariant with respect to $W\left(g_{c}, h_{c}\right)$.

We shall obtain these two results by a method that is somewhat different from Harish-Chandra's. We shall prove (3) first when $G$ is compact and then extend it for noncompact $G$ by the "unitarian trick". The second result is then deduced from formula (3). The proof of (3) for compact $G$ is quite simple and goes as follows. By invariant integration and the Weyl character formula, we obtain explicit formulae for a class of invariant eigenfunctions on 9 , the formula (3) applied to these eigenfunctions now determines $\delta_{b}^{\prime}(\partial(p))$ uniquely.

2. Case when $G$ is compact. We now assume that $G$ is compact and simply connected. Let $B$ be the analytic subgroup of $G$ corresponding to $\bar{G}, B$ is a maximal torus of $G$ and $G=B^{G}$. Exp is a homomorphism of $\mathscr{G}$ onto $B$. Let $\hat{B}$ be the character group of $B$. If $\xi \in \hat{B}, \xi \circ \exp$ is a character of $\zeta$, and so there is a $C$-linear function $\lambda$ on $\xi_{c}$, which takes pure imaginary values on $\bar{G}$, such that $\xi \circ \exp =e^{\lambda} . \lambda$ is uniquely determined by $\xi$, and we write $\xi=\xi_{\lambda}$. Thus $\xi_{\lambda}(\exp H)=e^{\lambda(H)}(H \in \mathfrak{G})$.

Let $\mathcal{L}$ be the set of all linear functions on $\mathscr{G}_{c}$ with the property that $e^{\lambda}=$ $\xi \circ \exp$ for some $\xi \in \hat{B} . \mathcal{L}$ is an additive subgroup of $\xi_{c}^{*}$ and the correspondence $\lambda \rightarrow \xi_{\lambda}(\lambda \in \mathscr{L})$ is an is omorphism of $\mathcal{Q}$ onto $\hat{B}$. Since $G$ is simply connected, $\mathcal{Q}$ is precisely the set of all integral linear functions on $\mathscr{G}_{c}[6]$. If $\alpha$ is a root of $\left(g_{c}, g_{c}\right)$, then $a \in \mathcal{\&}$. Now $B$ is the centralizer of $G$ in $G, \operatorname{Ad}(B)$ leaves the root-subspaces corresponding to $a$ invariant, and we have $X_{a}^{b}=\xi_{a}(b) X_{a}(b \in B$, $\left.X_{a} \in g_{a}\right)$. Define $\delta=1 / 2 \Sigma_{a \in P} a ; \delta$ is integral, and so $\delta \in \mathcal{L}$. Let $\Delta=$ $\xi_{-\delta} \Pi_{a \epsilon P}\left(\xi_{a}-1\right) . \Delta$ is a finite sum of characters of $B$. If we write $\epsilon(s)=\operatorname{det}(s)$ for $s \in W=W\left(g_{c}, g_{c}\right)$, it is known that $\Delta=\Sigma_{s \in W} \epsilon(s) \xi_{s a}$. Denote by $B^{\prime}$ the set of $b \in B$ such that $\Delta(b) \neq 0$, i.e. $\xi_{a}(b) \neq 1$ for all $a \in P$.

Let $\mathcal{D}_{p}^{+}$be the set of all dominant integral linear functions on $\xi_{c}$. For any $\lambda \in \mathfrak{D}_{p}^{+}$, let $\pi_{\lambda}$ be the irreducible representation of $g_{c}$ with highest weight $\lambda$. Since $G$ is simply connected, $\pi_{\lambda}$ lifts to a representation of $G$, denoted by $\pi_{\lambda}$ again. 
Let $\psi_{\lambda}(x)=\operatorname{tr} \pi_{\lambda}(x), x \in G$. We then have the following formula of Weyl.

$$
\psi_{\lambda}(b)=\sum_{s \in W} \epsilon(s) \xi_{s(\lambda+\delta)}(b) / \Delta(b) \quad\left(b \in B^{\prime}\right) .
$$

In particular, for $H \in \mathfrak{G}$ tor which $\exp H \in B^{\prime}$,

$$
\operatorname{tr} \pi_{\lambda}(\exp H)=\sum_{s \in W} \epsilon(s) e^{s(\lambda+\delta)(H)} / \prod_{\alpha \in P}\left(e^{a(H) / 2}-e^{-a(H) / 2}\right)
$$

and

$$
\operatorname{dim} \pi_{\lambda}=\prod_{a \in P}\langle\lambda+\delta, \alpha\rangle / \prod_{\alpha \in P}\langle\delta, \alpha\rangle
$$

Let $\mathcal{G}$ be the universal enveloping algebra of $g_{c}$ and $\mathcal{Z}$ the center of $\mathcal{G}$. By Schurr's lemma there is a homomorphism $\chi_{\lambda}$ (called the infinitesimal character of $\pi_{\lambda}$ ) of $\mathcal{Z}$ into $\mathrm{C}$, such that $\pi_{\lambda}(z)=\chi_{\lambda}(z) \cdot 1$ for all $z \in \mathcal{Z}$. Let $\mathcal{H}$ be the subalgebra of $\mathcal{G}_{g}$ generated by 1 and $\mathscr{G}_{c}$, and let $\mathcal{P}$ be the left ideal $\Sigma_{a \in P} \mathcal{G}_{\alpha}$. Then it can be shown that for each $z \in \mathcal{E}$, there is a unique element $\beta_{p}(z) \in \mathcal{H}$ such that $z-\beta_{p}(z) \in \mathscr{P}$. The map $\beta_{p}: z \rightarrow \beta_{p}(z)$ is a homomorphism of $\mathcal{Z}$ onto $\mathcal{H}$, and (cf. Harish-Chandra [4])

$$
\chi_{\lambda}(z)=\beta_{p}(z)(\lambda) \quad(z \in \mathscr{Z}) .
$$

Let $U$ be a $G$-invariant open subset of $g$ and $f \in C^{\infty}(U)$. We say $f$ is an invariant eigenfunction on $g$, if $f$ is $G$-invariant, and for each $p \in I_{s}\left(g_{c}\right)$, there is a $\chi(p) \in \mathbf{C}$ such that

$$
\partial(p) f=\chi(p) f \text { on } U \text {. }
$$

$\chi$ is uniquely determined if $f \neq 0$ and $\chi(p \rightarrow \chi(p))$ is a homomorphism of $I_{s}\left(g_{c}\right)$ into $C$.

Lemma 2.1. Let $d x$ be the normalized Haar measure on $G$, and $X^{\prime} \in g_{c}$. Define

$$
f_{X^{\prime}}(X)=f\left(X^{\prime}: X\right)=\int_{G} \exp B\left(X^{x}, X^{\prime}\right) d x \quad(X \in g)
$$

Then $f_{X^{\prime}}$ is an invariant eigenfunction on $g$, and

$$
\partial(p) f_{X^{\prime}}=\tilde{p}\left(X^{\prime}\right) f_{X^{\prime}} \quad\left(p \in I_{s}\left(g_{c}\right)\right)
$$

where $p \rightarrow \tilde{p}$ is the isomorphism of $S\left(g_{c}\right)$ onto $P\left(g_{c}\right)$ induced by the CartanKilling form. 
Proof. Let $\phi(x, X)=\exp B\left(X^{x}, X^{\prime}\right), x \in G, X \in g_{c}$. Then $\phi \in C^{\infty}(G \times g)$ and as $G$ is compact, the integral defined by $f_{X},(X)$ exists. The function $X \rightarrow f_{X},(X)$ is of class $C^{\infty}$ on $g$ and we can differentiate under the integral sign. If $p \in S\left(g_{c}\right)$,

$$
f_{X^{\prime}}(X ; \partial(p))=\int_{G} \phi(x: X ; \partial(p)) d x \quad(X \in g) .
$$

Let $\omega \in g_{c}^{*}$. Then using the natural identification of $S\left(g_{c}\right)$ with $P\left(g_{c}^{*}\right)$, we obtain $\partial(p) e^{\omega}=p(\omega) e^{\omega}$. If $Y \in g_{c}$ and $\omega$ is the linear function $X \rightarrow B(X, Y)$, then $p(\omega)=\tilde{p}(Y)$. From these and the fact that $\phi(x, X)=\exp B\left(X, X^{\prime} x^{-1}\right)$, we get, for all $x \in G, X \in g$,

$$
\phi(x, X ; \partial(p))=\tilde{p}\left(X^{\prime x^{-1}}\right) \phi(x, X) \text {. }
$$

If we assume $p \in I_{s}\left(g_{c}\right)$, we obtain $\partial(p) f_{X^{\prime}}=\tilde{p}\left(X^{\prime}\right) f_{X^{\prime}}$. Invariance of ' $f_{X^{\prime}}$ follows from translation invariance of $d x$.

Our aim in the rest of this section is to evaluate the $f_{X^{\prime}}$. This requires some preparation. For any subspace $a$ of $g_{c}$, we shall identify $S(a)$ with the subalgebra of $S\left(g_{c}\right)$ generated by 1 and $a$. For any $n \geq 0$, write $S_{n}\left(g_{c}\right)$ for the subspace of $S\left(g_{c}\right)$ spanned by homogeneous elements of degree $n$. Set $S^{(n)}\left(g_{c}\right)=$ $\Sigma_{0 \leq r \leq n} S_{r}\left(g_{c}\right)$. Let $\tilde{\lambda}$ be the customary symmetrizer map of $S\left(g_{c}\right)$ onto $\mathcal{G}_{\text {. Since }}$ $\tilde{\lambda}\left(p^{x}\right)=\tilde{\lambda}(p)^{x}\left(p \in S\left(g_{c}\right), x \in G\right), \tilde{\lambda}$ is a linear bijection of $I_{s}\left(g_{c}\right)$ onto $\mathcal{Z}$. It is also known to be a linear bijection of $S^{(n)}\left(g_{c}\right)$ onto the subspace $\mathcal{G}^{(n)}$ of $\mathcal{G}$ spanned by 1 and elements of the form $X_{1} \ldots X_{r}\left(1 \leq r \leq n, X_{i} \in g_{c}\right)$.

Lemma 2.2. Let 8 be the subspace of $g_{c}$ spanned by $X_{a}, a \in \Delta$. Then $S\left(g_{c}\right)=S\left(G_{c}\right)+S\left(g_{c}\right) \delta_{\text {. F }}$ For any $p \in S\left(g_{c}\right)$, let $p_{b}$ denote the unique element of $S\left(g_{c}\right)$ such that $p-p_{\mathfrak{b}} \in S\left(g_{c}\right) \delta_{\text {. Then }} p \rightarrow p_{\mathfrak{b}}$ is an isomorphism of $I_{s}\left(g_{c}\right)$ onto $I_{s}\left(G_{c}\right)$. If $p \in I_{s}\left(g_{c}\right) \cap S_{n}\left(g_{c}\right)$ then $p_{b} \in I_{s}\left(G_{c}\right) \cap S_{n}\left(G_{c}\right)$.

Proof. The statements are all consequences of the theorem of Chevalley mentioned earlier.

Put $\overline{\mathcal{H}}=\left\{a \in \mathcal{H}: a^{s}=a\right.$ for all $\left.s \in W\right\}$. The map $\tilde{\lambda}$, by restriction, induces an isomorphism of $S\left(\mathscr{G}_{c}\right)$ onto $\mathcal{H}$, and $I_{s}\left(\mathscr{G}_{c}\right)$ onto $\overline{\mathcal{H}}$. Let $\mathcal{H}_{n}=\tilde{\lambda}\left(S_{n}\left(\mathscr{G}_{c}\right)\right)$. $\mathcal{H}$ is the direct sum of $\mathcal{H}_{n}$ 's, and for any $v \in \mathcal{H}$, we write $v_{n}$ for its component in $\mathcal{H}_{n}$. Put $\mathcal{H}^{(n)}=\Sigma_{0 \leq r \leq n} \mathcal{H}_{r}$. Then

Lemma 2.3. Let $p \in I_{s}\left(g_{c}\right) \cap S_{n}\left(g_{c}\right)$. Then $\tilde{\lambda}(p) \in \mathcal{Z} \cap \varrho^{(n)}, \beta_{p}(\tilde{\lambda}(p)) \in \mathcal{H}^{(n)}$, $\beta_{p}(\tilde{\lambda}(p))_{n}=\tilde{\lambda}\left(P_{b}\right)$.

Proof. Let $\left\{H_{1}, \ldots, H_{l}\right\}$ be a basis for $K_{c}$ and $P=\left\{a_{1}, \ldots, a_{d}\right\}$. Let $(q)=\left(q_{1}, \ldots, q_{d}\right),(c)=\left(c_{1}, \ldots, c_{l}\right),(r)=\left(r_{1}, \cdots, r_{d}\right)$, where $q_{i}, c_{j}$, and $r_{k}$ are integers $\geq 0$. Define 


$$
M((q),(c),(r))=X_{-a_{1}}^{q_{1}} \cdots X_{-a_{d}}^{q_{d}} \cdot H_{1}^{c} \cdots H_{l}^{c} \cdot X_{a_{1}}^{r_{1}} \cdots X_{a_{d}}^{r}
$$

$p \in I_{s}\left(g_{c}\right)$ if and only if it is a linear combination of $M((q),(\epsilon),(r))$ for which $\sum_{i=1}^{d}\left(r_{i}-q_{i}\right) a_{i}=0$. Hence we can find constants $A((q),(c),(r))$ (all but finitely many of them zero) such that

$$
p=p_{\mathfrak{h}}+\sum_{\mathbf{\Omega}} A((q),(c),(r)) M((q),(c),(r))
$$

where the sum is over the set $\Omega$ of all $((q),(c),(r))$ for which $\sum_{i=1}^{d}\left(r_{i}-q_{i}\right) \alpha_{i}=0$, $\Sigma_{i} r_{i}>0, \sum_{i} q_{i}>0$ and $\sum_{i=1}^{d}\left(r_{i}+q_{i}\right)=n$.

On the other hand by considering $M((q),(c),(r))$ as an element of $\mathcal{G}$, we can show that if $z \in \mathscr{Z} \cap \mathrm{G}^{(n)}$, then $\beta_{p}(z) \in \mathcal{H}^{(n)}$. Let $z=\tilde{\lambda}(p)$, then using (8) and the result that $\tilde{\lambda}(M((q),(c),(r))) \in \mathscr{P}+\mathcal{S}^{(n-1)}$, and the fact that $\beta_{p}$ maps $\varrho^{(n-1)}$ into $\mathcal{H}^{(n-1)}$ we conclude

$$
\beta_{p}(z) \in \tilde{\lambda}\left(p_{\mathfrak{b}}\right)+\mathcal{H}^{(n-1)} .
$$

But $\tilde{\lambda}\left(p_{b}\right) \in \mathcal{H}_{n}$. Hence (9) implies the result.

Consider now $u \in S_{n}\left(g_{c}\right)$, then $p=\int_{G} u^{x} d x$ is a well-defined element of $I_{s}\left(g_{c}\right) \cap S_{n}\left(g_{c}\right)$. Also forming $\int_{G} \tilde{\lambda}(u)^{x} d x$, we obtain an element $z$ of $\mathcal{Z} \cap \mathcal{G}^{(n)}$. Since $\tilde{\lambda}\left(u^{x}\right)=\tilde{\lambda}(u)^{x}, x \in G$, we have $z=\tilde{\lambda}(p)$. Lemma 2.3 , now shows that $\beta_{p}(z)_{n}=\tilde{\lambda}\left(p_{b}\right)$. Taking $u=H^{n}$ for $H \in \mathscr{K}_{c}$ we get

Lemma 2.4. Let $n \geq 0$. Then for any $H \in \mathscr{G}_{c}$

$$
\zeta_{H, n}=\int_{G}\left(H^{n}\right)^{x} d x
$$

is a well-defined element of $I_{s}\left(g_{c}\right) \cap S_{n}\left(g_{c}\right)$ while considering the same integral

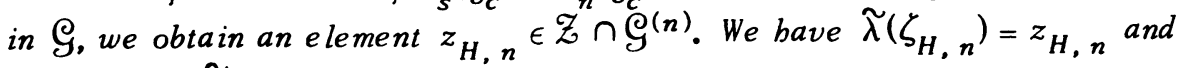
$\beta_{p}\left(z_{H, n}\right)_{n}=\tilde{\lambda}\left(\left.\zeta_{H, n}\right|_{b}\right)$.

Corollary 2.5. Let $\mu \rightarrow H_{\mu}^{\prime}$ be the is omorphism of $\mathscr{G}_{c}^{*}$ with $\mathscr{G}_{c}$ induced by the Cartan-Killing form. Then for any $H \in \mathfrak{G}_{c}, n \geq 0$ and $\mu \in \mathfrak{G}_{c}^{*}$,

$$
\int_{G} B\left(H^{x}, H_{\mu}^{\prime}\right)^{n} d x=\beta_{p}\left(z_{H, n}\right)(\mu) .
$$

Proof. From Lemma 2.4 , we have $\beta_{p}\left(z_{H, n}\right)(\mu)=\tilde{\lambda}\left(\left.\zeta_{H, n}\right|_{\mathfrak{h}}\right)(\mu)$ for $\mu \in \zeta_{c}^{*}$. From isomorphism $p \rightarrow \tilde{p}$ of $S\left(g_{c}\right)$ with $P\left(g_{c}\right)$ and the definition of $\zeta_{H, n}$, we obtain

$$
\zeta_{H, n}(Y)=\int_{G} B\left(H^{x}, Y\right)^{n} d x \quad\left(H \in \mathscr{G}, Y \in g_{c}\right)
$$

So for $\mu \in \mathfrak{G}_{c}^{*}$ 


$$
\int_{G} B\left(H^{x}, H_{\mu}^{\prime}\right) d x=\widetilde{\zeta}_{H, n}\left(H_{\mu}^{\prime}\right)
$$

On the other hand, the map $p \rightarrow p_{\mathfrak{h}}$ corresponds, via the isomorphism $p \rightarrow \widetilde{p}$, to the restriction map $\left.\tilde{\sim} \longrightarrow \widetilde{p}\right|_{\mathfrak{h} c}$ of $P\left(g_{c}\right)$ onto $P\left(\xi_{c}\right)$ so $\left.\widetilde{\zeta}_{H_{n},}\right|_{\mathfrak{h}}=\left(\left.\zeta_{H_{n} n}\right|_{\mathfrak{h}}\right)^{\sim}$, from which we get $\widetilde{\zeta}_{H, n}\left(H_{\mu}^{\prime}\right)=\left(\left.\zeta_{H, n}\right|_{\mathfrak{b}}\right)(\mu), \mu \in \zeta_{c}^{*}$. Since $\tilde{\lambda}\left(\bar{H}^{n}\right)=\bar{H}^{n}, \vec{H} \in \mathfrak{h}_{c}$, $\tilde{\lambda}\left(\left.\zeta_{H, n}\right|_{\mathfrak{h}_{c}}\right)(\mu)=\left(\left.\dot{\zeta}_{H, n}\right|_{\mathfrak{h}_{c}}\right)(\mu)$.

We are now in a position to obtain a formula for $f_{X}$, defined in Lemma 2.1.

Theorem 2.6. Let $G$ be a compact and semisimple real Lie group, g its Lie algebra, $5 \subset g$ a Cartan subalgebra. $P$ a positive system of roots, $\pi=\Pi_{a \in P} a$, $\tilde{\omega}=\Pi_{a \in p} H_{a}^{\prime}$. Then for all $H, H^{\prime} \in \bar{G}$ we have

$$
\pi(H) \pi\left(H^{\prime}\right) \int_{G} \exp B\left(H^{x}, H^{\prime}\right) d x=\left(\prod_{a \in P}\langle\delta, a\rangle\right) \sum_{s \in W} \epsilon(s) e^{B\left(s H, H^{\prime}\right)} .
$$

Proof. We have $\exp B(X, Y)=\Sigma_{n \geq 0} B(X, Y)^{n} / n !$. Since $\left|B\left(X^{x}, Y\right)\right|$ is bounded as $x$ varies in $G$, we can write

$$
\int_{G} \exp B\left(H^{x}, H^{\prime}\right) d x=\sum_{n \geq 0} \frac{1}{n !} \int_{G} B\left(H^{x}, H^{\prime}\right)^{n} d x .
$$

Upon replacing $H^{\prime}$ by $H_{\mu}^{\prime}\left(\mu \in \mathfrak{K}_{c}^{*}\right)$ in above and using Corollary 2.5, we get

$$
\int_{G} \exp B\left(H^{x}, H_{\mu}^{\prime}\right) d x=\sum_{n \geq 0} \frac{1}{n !} \beta_{p}\left(z_{H, n}\right)(\mu) \text {. }
$$

For $\lambda \in \mathscr{D}_{p}^{+}$, let $\pi$ be the irreducible representation of $g_{c}$ and $G$ with highest weight $\lambda$. From (7) we obtain

Hence, as $z_{H, n} \in Z$

$$
\beta_{p}\left(z_{H, n}\right)(\lambda)=\chi_{\lambda}\left(z_{H, n}\right) .
$$

$$
\operatorname{tr} \pi_{\lambda}\left(z_{H, n}\right)=\left(\operatorname{dim} \pi_{\lambda}\right) \chi_{\lambda}\left(z_{H, n}\right)=\left(\operatorname{dim} \pi_{\lambda}\right) \beta_{p}\left(z_{H, n}\right)(\lambda) .
$$

Using (6) in above we get

$$
\tilde{\omega}(\lambda+\delta) \beta_{p}\left(z_{H, n}\right)(\lambda)=\prod_{a \in p}\langle\delta, \alpha\rangle \operatorname{tr} \pi_{\lambda}\left(z_{H, n}\right) .
$$

But from the definition of $z_{H, n}$,

$$
\operatorname{tr} \pi_{\lambda}\left(z_{H, n}\right)=\int_{G} \operatorname{tr} \pi_{\lambda}\left(H^{n}\right)^{x} d x=\operatorname{tr} \pi_{\lambda}\left(H^{n}\right) .
$$

Now, for $t \in \mathbf{R}$,

$$
\pi_{\lambda}(\exp t H)=e^{t \pi_{\lambda}(H)}=\sum_{n \geq 0} \frac{t^{n} \pi_{\lambda}\left(H^{n}\right)}{n !} .
$$


So

$$
\operatorname{tr} \pi_{\lambda}(\exp t H)=\sum_{n \geq 0} \operatorname{tr} \pi_{\lambda}\left(H^{n}\right) \frac{t^{n}}{n !} .
$$

From Weyl's character formula (5)

$$
\prod_{a \in P}\left(e^{t a(H) / 2}-e^{-t a(H) / 2}\right) \operatorname{tr} \pi_{\lambda}(\exp t H)=\sum_{s \in W} \epsilon(s) e^{t s(\lambda+\delta)(H)}, \quad t \in \mathbf{R}, H \in \mathscr{G} .
$$

Using above formula in (11) we conclude

$$
\prod_{a \in P}\left(e^{t a(H) / 2}-e^{-t a(H) / 2}\right) \sum_{n \geq 0} \frac{\operatorname{tr} \pi_{\lambda}\left(H^{n}\right) t^{n}}{n !}=\sum_{s \in W} \epsilon(s) e^{t s(\lambda+\delta)(H)} .
$$

Upon expanding $\Pi_{a \in P}\left(e^{t a(H) / 2}-e^{-t a(H) / 2}\right)$ and letting $d=[P]$ we get

$$
\prod_{a \in P}\left(e^{t a(H) / 2}-e^{-t a(H) / 2}\right)=t^{d} \pi(H) \sum_{m \geq 0} q_{m}^{\prime} t^{2 m} .
$$

For sufficiently small $|t|$ above converges, hence from (12)

$$
t^{d} \pi(H) \sum_{n \geq 0} \frac{\operatorname{tr} \pi_{\lambda}\left(H^{n}\right)}{n !} t^{n}=\left[\sum_{m>0} q_{m}^{\prime} t^{2 m}\right]^{-1} \sum_{s \in W} \epsilon(s) e^{t s(\lambda+\delta)(H)} .
$$

Since $q_{0}^{\prime}=1$, for small $|t|$, we have $\left(\Sigma_{m} q_{m}^{\prime} t^{2 m}\right)^{-1}=\Sigma_{m \geq 0} q_{m} t^{2 m}$. Using this in above formula, expanding the right-hand side, and equating the coefficients of $t^{n+d}$, we get

$$
\frac{\pi(H)}{n !} \operatorname{tr} \pi_{\lambda}\left(H^{n}\right)=\frac{\sum_{s \in W} \epsilon(s)(s(\lambda+\delta)(H))^{n+d}}{(n+d) !}+q_{1} \frac{\sum_{s \in W} \epsilon(s)(s(\lambda+\delta)(H))^{n+d-2}}{(n+d-2) !}+\cdots
$$

Combining this with (10) we get

$$
\begin{aligned}
\left(\prod_{a \in P}\langle\delta, a\rangle\right)^{-1} & \frac{\pi(H)}{n !} \tilde{\omega}(\lambda+\delta) \beta_{p}\left(z_{H, n}\right)(\lambda) \\
& =\frac{\sum_{s \in W} \epsilon(s)(s(\lambda+\delta)(H))^{n+d}}{(n+d) !}+q_{1} \frac{\sum_{s \in W} \epsilon(s)(s(\lambda+\delta)(H))^{n+d-2}}{(n+d-2) !}+\cdots
\end{aligned}
$$

$q_{i}$ 's are independent of $\lambda$, and since above is true for $\lambda \in \mathfrak{D}_{p}^{+}$, it is true for $\lambda \in \mathfrak{G}_{c}^{*}$. In above formula we equate components which are homogeneous polynomials of degree $(n+d)$ in $\lambda$ :

$$
\frac{\pi(H)}{n !} \tilde{\omega}(\lambda) \beta_{p}\left(z_{H, n}\right)(\lambda)=\prod_{a \in P}\langle\lambda, a\rangle \frac{\sum_{s \in W} \epsilon(s)(s \lambda(H))^{n+d}}{(n+d) !} .
$$

Substituting above in 


$$
\int_{G} \exp B\left(H^{x}, H_{\mu}^{\prime}\right) d x=\sum_{n \geq 0} \frac{1}{n !} \beta_{p}\left(z_{H, n}\right)(\mu)
$$

obtained earlier we conclude

$$
\begin{aligned}
\pi(H) \tilde{\omega}(\mu) & \int_{G} \exp B\left(H^{x}, H_{\mu}^{\prime}\right) d x \\
= & \left(\prod_{a \in p}\langle\delta, a\rangle\right) \sum_{m \geq d} \frac{1}{m !} \sum_{s \in W} \epsilon(s)((s \lambda)(H))^{m} .
\end{aligned}
$$

For $p \geq 0$ define $f_{p}(\lambda)=\Sigma_{s \in W^{\epsilon}}(s)((s \lambda)(H))^{p}, \lambda \in \bar{F}_{c}^{*} \cdot f_{p}$ is homogeneous of degree $p$ and is skew symmetric. It follows [1] that $f_{p}=0, p<d$. In view of above, we can rewrite (13) as

$$
\begin{aligned}
& \pi(H) \tilde{\omega}(\mu) \int_{G} \exp B\left(H^{x}, H_{\mu}^{\prime}\right) d x \\
& =\left(\prod_{a \in p}\langle\delta, a\rangle\right) \sum_{m \geq 0} \frac{1}{m !} \sum_{s \in W} \epsilon(s)((s \mu)(H))^{m}=\left(\prod_{a \in P}\langle\delta, a\rangle\right) \sum_{s \in W} \epsilon(s) e^{(s \mu)(H)} .
\end{aligned}
$$

Replacing $H_{\mu}^{\prime}$ by $H^{\prime}$, and noting that $\tilde{\omega}(\mu)=\pi\left(H_{\mu}^{\prime}\right)$ and $(s \mu)(H)=B\left(s H, H_{\mu}^{\prime}\right)$ we obtain the result.

From this theorem we obtain immediately the sought-for formulae for the eigenfunctions $f_{X}$.

Theorem 2.7. Let $f_{X}$, be defined as in Lemma 2.1, then

$$
f_{H^{\prime}}(H)=\left(\prod_{a \in P}\langle\delta, a\rangle\right) \frac{\sum_{s \in W} \epsilon(s) e^{B\left(s H, H^{\prime}\right)}}{\pi(H) \pi\left(H^{\prime}\right)} \quad\left(H, H^{\prime} \in \mathscr{G}^{\prime}\right) .
$$

3. The explicit calculation of $\delta_{b}^{\prime}(\partial(p))\left(p \in I_{s}\left(g_{c}\right)\right)$. We shall now prove (3), for the case $G$ is compact. Let $g$ be of compact type and define

$$
\delta_{\mathfrak{h}}(D)=\pi \circ \delta_{\mathfrak{h}}^{\prime}(D) \circ \pi^{-1} \quad(D \in \operatorname{Diff}(U)) .
$$

Lemma 3.1. Let $E \in \operatorname{Diff}\left(G^{\prime}\right)$ such that $E g_{H^{\prime}}=0$ on $\zeta^{\prime}$ where $g_{H^{\prime}}(H)=$ $\Sigma_{s \in W^{f}}(s) e^{B\left(s H, H^{\prime}\right)}\left(H, H^{\prime} \in G^{\prime}\right) ;$ then $E \equiv 0$.

Proof. Choose $q_{1}, \cdots, q_{k}$ linearly independent in $S\left(\mathscr{G}_{c}\right)$; then $E=$ $\sum_{i=1}^{k} g_{i} \partial\left(q_{i}\right), g_{i} \in C^{\infty}\left(\zeta^{\prime}\right) . E g_{H^{\prime}}=0$ implies

$$
\sum_{i=1}^{k} \sum_{s \in W} \epsilon(s)_{g_{i}}(H)_{q_{i}}^{\sim}\left(s H^{\prime}\right) e^{B\left(s H, H^{\prime}\right)} \equiv 0 \quad\left(H, H^{\prime} \in E^{\prime}\right)
$$


Fix $H \in G^{\prime}$, then the points $s H, s \in W$, are distinct (Chevalley). Thus $H^{\prime} \rightarrow$ $B\left(s H, H^{\prime}\right)$ are distinct linear forms. From a result of [3] and (16) we conclude

$$
\sum_{i=1}^{k} \epsilon(s) g_{i}(H) \tilde{q}_{i}^{s}\left(H^{\prime}\right) \equiv 0 \quad\left(H^{\prime} \in \xi\right) \text {. }
$$

Taking $s=1$ and using the linear independence of $q_{i}$ 's we obtain $g_{i}(H)=0$, $1 \leq i \leq k$. Therefore $E \equiv 0$.

Lemma 3.2. For $p \in I_{s}\left(g_{c}\right), \delta_{\mathfrak{b}}(\partial(p))=\partial\left(p_{\mathfrak{b}}\right)$.

Proof. If $g$ is a function on $\left(G^{\prime}\right)^{G}$, let $\bar{g}=\left.g\right|_{\mathfrak{h}^{\prime}}$. From (14) we get

$$
\bar{f}_{H^{\prime}}\left(H ; \pi^{-1} \circ \partial\left(p_{\mathfrak{h}}\right) \circ \pi\right)=\tilde{p}\left(H^{\prime}\right) \bar{f}_{H^{\prime}}(H) .
$$

But Lemma 2.1 implies $\partial(p) f_{H^{\prime}}=\tilde{p}\left(H^{\prime}\right) f_{H^{\prime}}$ on $\mathscr{G}$. From the definition of radial component it follows that

$$
\bar{f}_{H^{\prime}}\left(H ; \delta_{\mathfrak{h}}^{\prime}(\partial(p))\right)=\tilde{p}\left(H^{\prime}\right) \bar{f}_{H^{\prime}}(H) \quad\left(H \in \mathscr{G}^{\prime}\right) .
$$

Above formulae along with (15) imply $\left(\partial\left(p_{\mathfrak{h}}\right)-\delta_{\mathfrak{h}}(\partial(p))\right) g_{H^{\prime}}=0$ on $\xi^{\prime}$ where $g_{H^{\prime}}(H)=\Sigma_{s \epsilon W^{\epsilon}}(s) e^{B\left(s H, H^{\prime}\right)}\left(H, H^{\prime} \in \xi\right)$. The result follows from Lemma 3.1.

We are now in a position to prove (3) for an arbitrary semisimple Lie algebra over R. Let $g$ be one such and $G$ a connected Lie group with Lie algebra.

Lemma 3.3. Let $p \in I_{s}\left(\xi_{c}\right)$, then $\delta_{\mathfrak{h}}^{\prime}(\partial(p))=\pi^{-1} \circ \partial\left(p_{\mathfrak{h}}\right) \circ \pi$ on $\mathfrak{G}^{\prime}$ if and only if $\partial\left(p_{\mathfrak{h}}\right)\left(\pi q_{\mathfrak{h}}\right)=\pi(\partial(p) q)_{\mathfrak{h}}$ on $\bar{G}_{c}$ for all $q \in I_{p}\left(g_{c}\right)$.

Proof. Let $E=\delta_{\mathfrak{h}}^{\prime}(\partial(p))-\pi^{-1} \circ \partial\left(p_{\mathfrak{h}}\right) \circ \pi$ and $\mathcal{Q}$ the algebra of all real-valued $G$-invariant polynomials on $g$. It can be shown(2) $E \equiv 0$ on $\mathscr{G}^{\prime}$ if and only if $E \bar{g}=0$ for all $g \in \mathbb{Q}$. Recall that $\bar{g}=\left.g\right|_{\mathfrak{h}}$. This is equivalent to $\pi^{-1} \circ \partial\left(p_{\mathfrak{h}}\right)\left(\pi q_{\mathfrak{h}}\right)=$ $\delta_{\mathfrak{b}}^{\prime}(\partial(p))_{q_{\mathfrak{b}}}$ on $\xi^{\prime}$ for all $q \in I_{p}\left(g_{c}\right)$. Using the definition of $\delta_{\mathfrak{b}}^{\prime}(\partial(p))$ and above, we get $\partial\left(p_{\mathfrak{h}}\right)\left(\pi q_{\mathfrak{h}}\right)=\pi\left(\partial(p)_{q}\right)_{\mathfrak{h}}$ on $\bar{G}^{\prime}$. Since we are dealing with polynomials this implies the result.

Theorem 3.4. Let $g$ be a real semisimple Lie algebra, $g_{c}$ its complexifica. tion. Let $p \in I_{s}\left(g_{c}\right)$; then $\delta_{b}(\partial(p))=\partial\left(p_{b}\right)$.

Proof. Let $U$ be a compact real form of $g_{c}$, and $\mathfrak{b}$ a Cartan subalgebra of it. Then $\mathfrak{b}_{c}=\mathbb{C} \cdot \mathfrak{b}$ is a Cartan subalgebra of $g_{c}$ and hence $\mathfrak{b}_{c}^{x}=\mathfrak{G}_{c}$ for some $x \in G_{c}$.

(2) It suffices to show $E_{H_{0}}=0$ for $H_{0} \in b^{\prime} . I_{p}\left(g_{c}\right)$ is finitely generated by $l$ algebraically independent homogeneous elements of positive degree. It can be shown that we can choose these generators so that their restrictions to are real-valued. Let $\left\{q_{1}, \cdots, q_{l}\right\}$ be one such set. Then by the Chevalley theorem $\left\{\bar{q}_{1}, \ldots, \bar{q}_{l}\right\}$ generares $I_{p}\left(b_{c}\right)$. A theorem of Shephard and Todd $[5]$ implies $\left\{\bar{q}_{1}, \ldots, \bar{q}_{l}\right\}$ forms a $C^{\infty}$ coordinate system around $H_{0}$. A straightforward argument now implies the claim. 
Let $\pi$ be the product of positive roots of $\left(g_{c}, b_{c}\right)$. We arrange matters so that $\pi=\pi_{b}^{x}$. By Lemma 3.2, $\delta_{b}^{\prime}(\partial(p))=\pi^{-1} \circ \partial\left(p_{b}\right) \circ \pi$ on $\mathfrak{b}^{\prime}$. By Lemma 3.3, $\partial\left(p_{b}\right)\left(\pi_{b} q_{b}\right)=\pi_{b}(\partial(p) q)_{b}$ on $b_{c}$. We then have

$$
\partial\left(p_{\mathfrak{b}}^{x}\right)\left(\pi_{\mathfrak{b}}^{x} q_{\mathfrak{b}}^{x}\right)=\pi_{\mathfrak{b}}^{x}\left((\partial(p) q)_{b}\right)^{x} .
$$

But $p_{\mathfrak{b}}^{x}=p_{\mathfrak{h}}, q_{\mathfrak{b}}^{x}=q_{\mathfrak{b}}$. So we have $\partial\left(p_{\mathfrak{h}}\right)\left(\pi q_{\mathfrak{h}}\right)=\pi\left(\partial(p) q_{\mathfrak{h}}\right.$. This proves the theorem in view of Lemma 3.3.

4. A theorem on $\delta_{\mathfrak{b}}^{\prime}(D)$ when $D \in \mathscr{D}\left(g_{c}\right)$. We now use the previous results to obtain the main theorem concerning $\delta_{b}^{\prime}(D), D \in \mathscr{D}\left(g_{c}\right)$ (cf. Harish-Chandra [2]). For any $p \in S\left(g_{c}\right)$ define $\mu_{p}(D)=\partial(p) \circ D-D \circ \partial(p)\left(D \in \operatorname{Diff}\left(\left(G^{\prime}\right)^{G}\right)\right) . \mu_{p}$ is a derivation of $\mathscr{D}\left(g_{c}\right)$. Define $\mu_{p}^{m}(D)=\mu\left(\mu_{p}^{m-1}(D)\right), m \geq 2$.

Lemma 4.1. Let $D \in \mathscr{D}\left(g_{c}\right), p \in S\left(g_{c}\right)$. Then there is an integer $m=m(p, D) \geq 0$ sucb that $\mu_{p}^{m}(D)=0$.

Proof. Since we can write $D=\sum_{i=1}^{s} p_{i} \partial\left(q_{i}\right), p_{i} \in P\left(g_{c}\right), q_{i} \in S\left(g_{c}\right)$ we may assume $D=p_{1} \partial\left(q_{1}\right)$. We assert $\mu_{p}(D)$ can be written as $\sum_{i=1}^{k} p_{i}^{\prime} \partial\left(q_{i}^{\prime}\right)$ with $\operatorname{deg} p_{i}^{\prime}$ $<\operatorname{deg} p_{1}$ for all $i$. Write $E=F \partial\left(q_{1}\right)$ where $F=\left[\partial(p), p_{1}\right]$. Direct calculations show that $F$ can be written as $\sum_{j=1}^{N} f_{j} \partial\left(g_{j}\right)$ where each $f_{j}$ is of the form $\partial\left(b_{j}\right) p_{1}$ for some $b_{j} \in P\left(g_{c}\right)$ which is homogeneous of positive degree. So $\operatorname{deg} f_{j}<\operatorname{deg} p_{1}$ for all $j$. Since $E=\Sigma f_{j} \partial\left(g_{j} q_{1}\right)$ the result follows by induction on $\max _{i} \operatorname{deg} p_{i}$.

Lemma 4.2. Let $U \subset \bar{G}$ can be a connected and open set, $f \in C^{\infty}(U)$. Suppose for every $p \in I_{s}^{+}\left(G_{c}\right)$, there is an integer $m=m(p)>0$ such that $\partial(p)^{m} f=0$ on $U$. Then $f$ is the restriction to $U$ of a polynomial on $\mathfrak{G}_{c}$.

Proof. Let $p_{1}, \cdots, p_{l}$ be homogeneous generators of $I_{s}\left(g_{c}\right)$. Then it can be seen that the assumption implies $\partial\left(p_{i}\right)^{m} f=0$ for some $m \geq 0,1 \leq i \leq l$. Let $d_{i}=$ $\operatorname{deg}\left(p_{i}\right)$ and $k=m\left(d_{1}+\cdots+d_{l}\right)$. For $H \in \bar{G}$ consider $Q(\bar{\zeta})=\Pi_{s \in W}\left(\zeta^{k}-(s H)^{k}\right)$. $Q(H)=0$, so $\partial(Q(H)) f=0$. Let $\omega=[W]$. Then

$$
Q(\zeta)=\left(\zeta^{k}\right)^{\omega}+\left(\zeta^{k}\right)^{\omega-1} Q_{1, H}+\cdots+Q_{\omega, H}
$$

where $Q_{j, H}$ is a homogeneous element of $I_{s}\left(\mathscr{G}_{c}\right)$ of degree $j k$. For fixed $j, Q_{j, H}$ is a linear combination of monomials $p_{1}^{a_{1}} \cdots p_{l}{ }^{a}$ with $d_{1} a_{1}+\cdots+d_{l} a_{l}=j k \geq$ $k=m\left(d_{1}+\cdots+d_{l}\right)$. Hence there is at least one $a_{i}>m$, so that $\partial\left(p_{1}^{a_{1}} \cdots p_{l}^{a_{l}}\right) f=0$. Thus $\partial\left(Q_{j, H}\right) f=0$. Hence $\partial\left(H^{k \omega}\right) f=0$. As $U$ is connected, this implies $f$ is a polynomial of degree $\leq l k \omega$.

Lemma 4.3. Let $U \subset \bar{g}$ be a connected open set, $E \in \operatorname{Diff}(U)$. Suppose for each $p \in I_{s}^{+}\left(G_{c}\right)$ there is an integer $m=m(p)>0$ such that $\mu_{p}^{m}(E)=0$. Then there 
is an $F \in \mathscr{D}(\xi)$ such that $E=F$ on $U$, i.e. $E$ bas polynomial coefficients.

Proof. Write $E=\Sigma_{i=1}^{N} f_{i} \partial\left(g_{i}\right), f_{i} \in C^{\infty}(U), g_{i} \in S\left(\mathscr{G}_{c}\right)$. We will show $f_{i} \in P\left(\mathscr{G}_{c}\right)$. We may assume $g_{i}$ are linearly independent, homogeneous and $\operatorname{deg} g_{1} \leq \operatorname{deg} g_{2}$ $\leq \cdots \leq \operatorname{deg} g_{N}$. Also we may assume $f_{i} \notin P\left(\mathfrak{G}_{c}\right)$. Let $d=\min _{i} \operatorname{deg} g_{i}$ and $s \geq 1$ such that $\operatorname{deg} g_{i}=d, 1 \leq i \leq s, \operatorname{deg} g_{i}>d, s<i \leq N$. Let $p \in I_{s}\left(G_{c}\right)$. It can be shown

$$
\mu_{p}^{m}(E)=\sum_{i=1}^{N} \sum_{r=0}^{m}(-1)^{r}\left(\begin{array}{l}
m \\
r
\end{array}\right)(\partial(p))^{m-r}\left(f_{i} \partial\left(g_{i}\right)\right) \partial(p)^{r} .
$$

Let $M$, be the operator of multiplication by $f$, then $\partial(p)^{m-r} M_{f_{i}}=\Sigma_{t} U_{i, t} \partial\left(V_{i, t}\right)$ where $U_{i, t} \in C^{\infty}(U)$ and $V_{i, t}$ homogeneous. We then get

$$
\partial(p)^{m-r}\left(f_{i} \partial\left(g_{i}\right)\right) \partial(p)^{r}=\sum_{t} U_{i, t} \partial\left(V_{i, t} g_{i} p^{r}\right) .
$$

If $r=0$ we can write $\partial(p)^{m} M_{f_{i}}=M_{\partial(p)^{m} f_{i}}+\Sigma_{t} U_{i, t} \partial\left(V_{i, t}\right)$ where the $V_{i, t}$ are homogeneous elements of strictly positive degree. We then have

$$
\partial(p)^{m}\left(f_{i} \partial\left(g_{i}\right)\right)=\sum_{t} U_{i, t} \partial\left(V_{i, t} g_{i}\right)
$$

It follows from the above two equations that

$$
\mu_{p}^{m}(E)=\sum_{i=1}^{s}\left(\partial(p)^{m} f_{i}\right) \partial\left(g_{i}\right)+\sum_{j=1}^{N n} f_{m} \partial\left(g_{m_{j}}\right)
$$

where $f_{m_{j}} \in C^{\infty}(U)$ and $g_{m_{j}}$ are homogeneous elements of $S\left(\xi_{c}\right)$ of degree $>d$. If we choose $m$ so that $\mu_{p}^{m}(E)=0$, then we conclude from above formula that $\partial(p)^{m} f_{i}=0,1 \leq i \leq s$. Lemma 4.2 now implies that $f_{i} \in P\left(\mathscr{G}_{c}\right)$, a contradiction.

Theorem 4.4. Let $D \in \mathscr{g}(g)$ and $\delta_{\mathfrak{h}}(D)=\pi \circ \delta_{\mathfrak{b}}^{\prime}(D) \circ \pi^{-1}$. There is a unique element of $\mathfrak{D}(\mathfrak{G})$ which coincides with $\delta_{\mathfrak{h}}(D)$ on $\xi^{\prime}$.

Proof. We need only to prove the existence. By Theorem 2.1, $\delta_{b}: D^{\prime} \rightarrow$ $\pi \circ \delta_{\mathfrak{b}}^{\prime}\left(D^{\prime}\right) \circ \pi^{-1}$ is a homomorphism of $g\left(\left(G^{\prime}\right)^{G}\right)$ into Diff $\left(G^{\prime}\right)$. Suppose now $p \in I_{s}\left(g_{c}\right)$. By Lemma 4.1, there is an integer $m \geq 0$ such that $\mu_{p}^{m}(D)=0$. So

$$
\sum_{r=0}^{m}(-1)^{r}\left(\begin{array}{l}
m \\
r
\end{array}\right) \partial(p)^{m-r} D \partial(p)^{r}=0 .
$$

Apply homomorphism $\delta_{\mathfrak{h}}$ to above and note that by Theorem $3.4, \quad \delta_{\mathfrak{h}}(\partial(q))=$ $\partial\left(q_{\mathfrak{h}}\right)\left(q \in I_{s}\left(g_{c}\right)\right)$ we will get

$$
\sum_{r=0}^{m}(-1)^{r}\left(\begin{array}{l}
m \\
r
\end{array}\right) \partial\left(p_{\mathfrak{h}}\right)^{m-r} \delta_{\mathfrak{h}}(D) \partial\left(p_{\mathfrak{h}}\right)^{r}=0 \quad \text { on } \xi^{\prime} .
$$

Comparing above with (i7) we get 


$$
\mu_{p_{\mathfrak{h}}}^{m}\left(\delta_{\mathfrak{h}}(D)\right)=0 \text { on } \mathfrak{G}^{\prime} \text {. }
$$

Let $U$ be a connected open set in $G^{\prime}$. Since $p \rightarrow p_{b}$ is an isomorphism of $I_{s}\left(g_{c}\right)$ onto $I_{s}\left(G_{c}\right)$, we conclude from above that given any $q \in I_{s}\left(G_{c}\right)$ which is homogeneous of positive degree, there is an integer $m=m(p)>0$ such that $\mu_{q}^{m}\left(\delta_{\mathfrak{h}}(D)\right)=0$ on $U$. By Lemma 4.3, we can find an $L \in \mathscr{D}(\xi)$ such that

$$
\text { - } L=\delta_{\mathfrak{h}}(D) \text { on } U \text {. }
$$

It can be shown [1] that $\delta_{\mathfrak{b}}^{\prime}(D)$ can be written as $\pi^{-N^{\prime}} \circ F$ for some integer $N^{\prime} \geq 0$ and $F \in \mathscr{D}(\xi)$. So we can write $\delta_{\mathfrak{h}}(D)$ as $\pi^{-N} \circ F$ for some $N \geq 0$ and $F \in \mathscr{D}(\mathfrak{G})$. Comparing this with (18) and noting a rational function on $\zeta_{c}$ is determined by its restriction on $U$, we conclude that $L=\delta_{\mathfrak{h}}(D)$ on $\xi^{\prime}$.

\section{REFERENCES}

1. Harish-Chandra, Differential operators on a semisimple Lie algebra, Amer. J. Math. 79 (1957), 87-120. MR 18, 809.

2. - Invariant differential operators and distributions on a semisimple Lie algebra, Amer. J. Math. 86 (1964), 534-564. MR 31 \# 4862a.

3. - The characters of semisimple Lie groups, Trans. Amer. Math. Soc. 83 (1956), 98-163. MR 18, 318.

4. - On some applications of the universal enveloping algebra of a semisimple Lie algebra, Trans. Amer. Math. Soc. 70 (1951), 28-96. MR 13, 428.

5. G. C. Shephard and J. A. Todd, Finite unitary reflection groups, Canad. J. Math. 6 (1964), 274-304. MR 15, 600.

6. Hermann Weyl, Selecta Hermann Weyl, Birkhäuser Verlag, Basel und Stuttgart, 1956. MR 17, 814.

DEPARTMENT OF MATHEMATICS, CARNEGIE-MELLON UNIVERSITY, PITTSBURGH, PENNSYLVANIA 15213

Current address: Department of Mathematics, University of Texas at Austin, Austin, Texas 78712 